\title{
Interactive Deconvolution with Error Analysis (IDEA) in astronomical imaging: Application to aberrated HST images on SN 1987A, M 87 and $3{\mathrm{C} 66 \mathrm{~B}^{\star}}^{\star}$
}

\author{
K. Bouyoucef, D. Fraix-Burnet ${ }^{\star \star}$, and S. Roques \\ Laboratoire d'Astrophysique de Toulouse, UMR 5572, GdR-PRC ISIS, Observatoire Midi-Pyrénées, 14 Avenue Edouard Belin, \\ 31400 Toulouse, France
}

Received March 14; accepted June 11, 1996

\begin{abstract}
The regularized reconstruction algorithm Interactive Deconvolution with Error Analysis (IDEA) is presented. One of its main advantages is to control the error propagation and to provide an estimation of the quality of the deconvolved image. IDEA is compared with two popular probabilistic methods: Richardson-Lucy and Maximum Entropy. These three methods are applied to Hubble Space Telescope aberrated images: SN 1987A and the jets of $\mathrm{M} 87$ and $3 \mathrm{C} 66 \mathrm{~B}$.
\end{abstract}

Key words: techniques: image processing supernovae: individual: SN 1987A — galaxies: jets galaxies: individual: M 87 - galaxies: individual: 3C 66B

\section{Introduction}

The Hubble Space Telescope (HST) observations, before the COSTAR correction, were strongly blurred by the spherical aberration of the primary mirror. In that case, conventional procedures in image restoration such as inverse filtering, matched filtering, etc., turned out to be unsuited to this problem.

The "new HST" now provides improved dynamic ranges, sensitivity to faint sources and increased resolution. However, the algorithms that were used on the old set of HST images are still relevant to the new instrument, since they are designed to perform better when applied to higher quality data.

\footnotetext{
* Based on observations with the NASA/ESA Hubble Space Telescope, obtained at the Space Telescope Science Institute, which is operated by the Association of Universities for Research in Astronomy, Inc., under NASA contract NAS5-26555.

** Present address: Laboratoire d'Astrophysique, URA 708, Observatoire de Grenoble, BP. 53X, 38041 Grenoble, France.
}

In Sect. 2, we remind the reader the general principles of the reconstruction algorithm IDEA (Interactive Deconvolution with Error Analysis) (Lannes et al. 1987ac). The deconvolution problem is stated in terms of weighted spectral interpolation and partial extrapolation. This operation is quantitatively related to the choice of a synthetic aperture which defines a limit in resolution. We propose variations on the general scheme by examining the contribution of Multiresolution Analysis (Mallat 1989). In Sect. 3, we compare our deterministic viewpoint with two probabilistic approaches: the Richardson-Lucy Method (RLM; Lucy 1974) and the Maximum Entropy Method (MEM; Narayan \& Nityananda 1986) applied to images of SN 1987A, M 87 and 3C 66B. Sect. 4 is devoted to an interpretation of the results and to an astrophysical discussion.

\section{Interactive deconvolution with error analysis}

The deconvolution problem is basically an ill-posed problem (Tikhonov \& Arsenin 1977): it does not fulfill the three Hadamard conditions of existence, uniqueness and stability of the solution. The last condition of stability may cause the main problems because if it is not fulfilled, a slight error in the data may lead to a very large error in the solution.

To circumvent the ill-conditionedness nature of this problem, one is led to postulate that the properties of the solution are not entirely contained in the equation to be solved. Therefore, one has to introduce a priori information on the solution to regularize the deconvolution process.

\subsection{IDEA - methodological principles}

To a first approximation, the experimental data $\phi_{\mathrm{i}}$ are related to the "original object" $\phi_{\mathrm{o}}-$ the intensity of the source at some high level of resolution - by an 
experimental Point-Spread Function (PSF) $\widehat{h}$ in the following way:

$\phi_{\mathrm{i}}(x)=\left(\phi_{\mathrm{o}} * \widehat{h}\right)(x)+e(x)$,

where $e$ is an additive term including random or systematic errors (i.e. errors on the determination of the PSF, linearity assumption, image sampling...) and signaluncorrelated random noise (telescope, detectors, atmosphere, guiding...). The support of $\phi_{\mathrm{o}}$ is contained in some finite region $V$ whose size and shape, determined in an interactive manner, will prove to play an essential role.

As the support $H$ of the transfer function $h$, Fourier transform of $\widehat{h}$, determines the experimental spatialfrequency aperture, one defines a centrosymmetric synthetic aperture $H_{\mathrm{r}}$, including $H$, and regularizing it. The choice of the diameter of this synthetic aperture defines the best compromise possible between the resolution to reach and the stability of the solution. Of course, it is preferable to give up trying to determine $\phi_{0}$ at its highest level of resolution, and one defines the "object to be reconstructed" $\phi_{\mathrm{s}}$ as a smoothed version of $\phi_{0}$ by a relation of the form $\widehat{\phi}_{\mathrm{s}}(u)=s(u) \widehat{\phi}_{0}(u)$, where $s(u)$ is a Prolate Spheroidal Function (Slepian \& Pollak 1961) whose energy is concentrated in $H_{\mathrm{r}}$.

The ratio of the amplitude spectrum of the image to the amplitude spectrum of the noise defines the pointwise signal-to-noise ratio SNR in the frequency space:

$\operatorname{SNR}(u)=\frac{\left|\widehat{\phi}_{\mathrm{i}}(u)\right|}{\sigma_{\mathrm{i}}(u)}$.

where $\sigma_{\mathrm{i}}(u)$ is an image-error bound such that $\mid \widehat{\phi}_{\mathrm{i}}(u)-$ $h(u) \widehat{\phi}_{0}(u) \mid \leq \sigma_{\mathrm{i}}(u)$.

Let us define a threshold value $\alpha_{\mathrm{t}}$ (in practice of the order of 1 or greater) under which SNR is considered as poor. In these conditions, one can give a first approximation $\widehat{\phi}_{\mathrm{t}}$ of the spectrum $\widehat{\phi}_{\mathrm{s}}$ of the object to be reconstructed:

$$
\widehat{\phi}_{\mathrm{t}}(u)=\left\{\begin{array}{cc}
s_{\mathrm{t}}(u) \widehat{\phi}_{\mathrm{i}}(u)=s(u) \frac{\widehat{\phi}_{\mathrm{i}}(u)}{h(u)} & \text { if } \operatorname{SNR}(u) \geq \alpha_{\mathrm{t}} \\
0 & \text { otherwise. }
\end{array}\right.
$$

Note that information contained in the spectrum of $\phi_{\mathrm{i}}$ when $\operatorname{SNR}(u)$ is less than $\alpha_{\mathrm{t}}$ is lost.

In the case of a deterministic procedure based on a least-squares minimization one defines the reconstructed object as the function which minimizes the functional:

$q(\phi)=\left\|g\left(\widehat{\phi}_{\mathrm{t}}-\widehat{\phi}\right)\right\|^{2}$,

where $g(u)$ is a weight function bounded by 1 , to be defined in relation to $\operatorname{SNR}(u)$ and $H_{\mathrm{r}}$. In particular, $g(u)=0$ in the parts of $H_{\mathrm{r}}$ where $\operatorname{SNR}(u) \leq \alpha_{\mathrm{t}}$ (characterizing the frequency gaps in $H_{\mathrm{r}}$ ), and $g(u)=1$ outside $H_{\mathrm{r}}$ (regularization principle). In the parts of $H_{\mathrm{r}}$ where the information must be taken into consideration, $g(u)$ is defined as an increasing function of $\operatorname{SNR}(u)$.

Before implementing a numerical iterative method for solving the problem, it is preferable to verify that the synthetic aperture (i.e. the resolution) has been well chosen, to avoid long and expensive computations leading in any case to an unstable solution.

The smallest eigenvalue $\mu$ of the imaging operator $v U g^{2} U^{*}$ conditions the stability of the reconstruction problem $\left(U\right.$ and $U^{*}$ are the direct and inverse Fourier Transform operators). It can be analytically estimated by examining some physical parameters of the problem: the functions $v$ and $w=1-g^{2}$, characteristic functions of $V$ and $H_{\mathrm{r}}$. Indeed, this eigenvalue is a function of the "interpolation parameter":

$\eta=\left(\int_{V} v(x) \mathrm{d} x\right)^{1 / 2}\left(\int_{H_{\mathrm{r}}} w(u) \mathrm{d} u\right)^{1 / 2}$

characterizing the amount of interpolation to be performed both in real and Fourier spaces. One has the following relation:

$\lambda(\eta)=\eta^{2} \sum_{m=0}^{\infty} k_{m} \eta^{2 m}$,

where the $k_{m}$ 's depend on $v$ and $w$ and are of the kind "moment of inertia" relatively to $H_{\mathrm{r}}$. This equation provides useful approximation to the minimum eigenvalue $\mu$ of the imaging operator occuring in the expression of an upper limit $\Theta$ of the quadratic reconstruction error:

$\frac{\|\Delta \phi\|}{\|\phi\|} \leq \Theta \quad$ where $\quad \Theta=\frac{1}{\sqrt{\mu}} \frac{\left\|g s_{\mathrm{t}} \sigma_{\mathrm{i}}\right\|}{\left\|\phi_{\mathrm{t}}\right\|}$.

Then, by suitably choosing $H_{\mathrm{r}}$ ( $V$ being quasi imposed), the size of the error can be acceptably small. So, we have an idea of the stability of the problem before its implementation. If this error remains too large, $\phi_{\mathrm{s}}$ must be redefined at a lower level of resolution by reducing $H_{\mathrm{r}}$. This operation, executable in an interactive manner, leads to a compromise between resolution and reliability. A more exhaustive error analysis can be conducted after the reconstruction process to obtain a better estimate of the upper-bound of the error (see Lannes et al. 1996).

\subsection{Contributions of multiresolution analysis}

The multiresolution analysis (MRA) provides a representation intermediate between the spatial and the Fourier 
ones: the data are represented as a superposition of wavelets at some scale level, each level being further decomposed into a lower scale level. The reader unfamiliar with this analysis is refered to Mallat (1989) or Daubechies (1992). Here, the MRA plays a decisive role in the control of the stability of the deconvolution (in particular when the field to resolution ratio increases).

Indeed, the MRA is very useful for the definition of the mathematical space in which the image has to be reconstructed: it can be decomposed in a collection of orthogonal sub-spaces corresponding to different resolutions. It is then usually easier to solve several sub-problems separately in each sub-space and to reconstruct the global solution afterwards. For instance, the deconvolution of SN 1987A makes use of a MRA for choosing the multiresolution support $V$ of the restored object (Fig. 1).

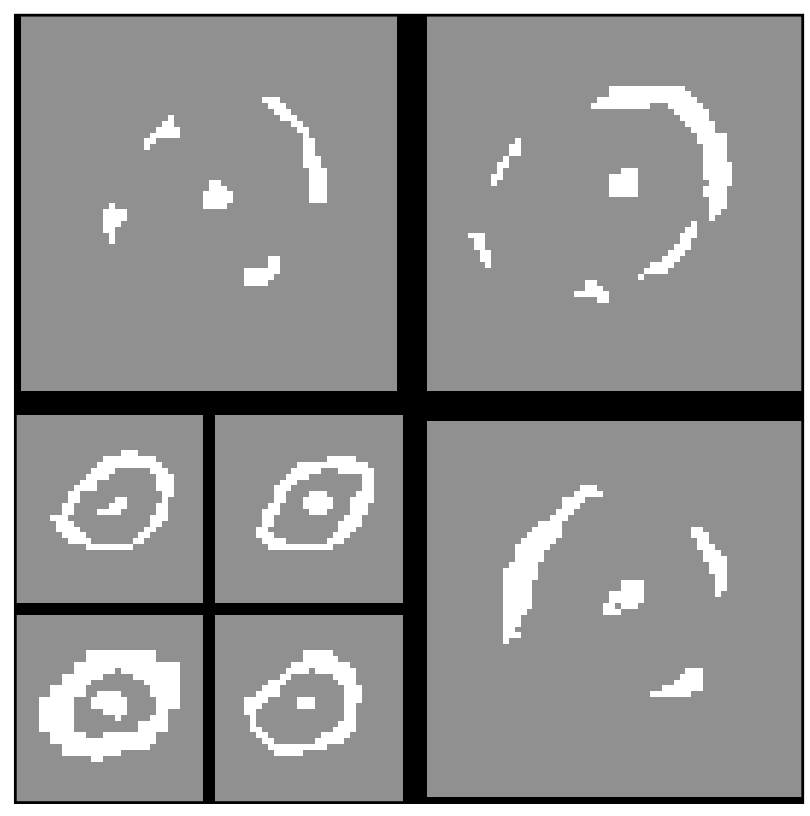

Fig. 1. Multiresolution support of SN 1987A

\section{Analysis of deconvolved HST images}

In this section, we compare the results obtained with the three deconvolution techniques (IDEA, RLM and MEM) using images obtained by the Hubble Space Telescope before the COSTAR correction.

First we present results on the supernova SN 1987A image because of its relatively simple structure (a bright core together with a well-delimited extended object, the ring). These results have been partially discussed in Bouyoucef et al. (1994). Second, the jet of M 87 is an example of a weak extended object aside a very bright unresolved source. And third the jet of $3 \mathrm{C} 66 \mathrm{~B}$ is a difficult case because of its very low signal-to-noise ratio.
All images are from the FOC with the $\mathrm{f} / 96$ mode (pixel size 0.'0224) and were retrieved from the HST archives. For RLM and MEM we used the softwares available in IRAF.

\section{1. $S N 1987 A$}

This $128 \times 128$ pixel image is a $822 \mathrm{~s}$ exposure through the F501N (OIII) filter obtained on August 24, 1990. It is described by Jakobsen et al. (1991) and a RLM deconvolution of this image is presented in Panagia et al. (1991). We use a PSF obtained on August 28, 1990 in the same telescope conditions as for SN 1987A.

We show in Fig. 2 the original image, and the solutions obtained with IDEA, RLM, and MEM. The RLM deconvolution is obtained after 50 iterations. For the MEM deconvolution, convergence is obtained with a uniform noise of 2.56, a Poisson noise coefficient of 0.35 , a quadratic noise coefficient of 0.03 (30 iterations); the result is smoothed by a Gaussian of FWHM equal to 2 pixels. For the IDEA deconvolution, we choose a gain in resolution of 1.9 yielding an upper limit of $17 \%$ to the quadratic error. The least-squares solution in IDEA is reached after 8 iterations. A multiresolution analysis has been used for the choice of the support (see Sect. 2).

The supernova star is anisotropic in all images: the RLM and MEM solutions show several spikes which are residuals of the PSF. On the IDEA solution this anisotropy is essentially an elongation at $\mathrm{PA} \simeq 45^{\circ}$ that could be physically related to the elliptical projected shape of an envelope around the supernova (see below).

A comparison of the profiles of the supernova between the different images is shown in Fig. 3. These profiles are obtained by plotting the intensity of each pixel as a function of its distance to the center. A polynomial fit of the profile of the PSF is presented in Fig. 3a, together with the profile of the faint star visible SE of the supernova. The first ring caused by the spherical aberration is clearly seen. The inner part of the PSF has FWHM $=0$ ".'06 corresponding to the unaberrated telescope. On the five other plots, the profile of the supernova on the raw and deconvolved images is compared to the profile of the PSF.

Clearly, the center of the supernova on the raw image has not the profile of the PSF, meaning that it is nonstellar (FWHM = 0.'13). This has already been found by Jakobsen et al. (1991), and its width indeed corresponds to the expected size of the envelope that was ejected in the 1987 explosion. Hence, the deconvolution should somehow preserve the profile of the center of the supernova. This is exactly the case with IDEA (FWHM = 0". 12, Fig. 3b) and MEM (FWHM = 0.'11, Fig. 3c); whereas the supernova on the RLM solution (Fig. 3d) has exactly the profile of the PSF, revealing over-resolution. One could argue that the number of iterations on the RLM solution is too high. We present results with 10 and 30 iterations (Fig. 3e and $\mathrm{f})$ : the supernova is already over-resolved at 10 iterations. We also note that the smooth shape of the core after IDEA 

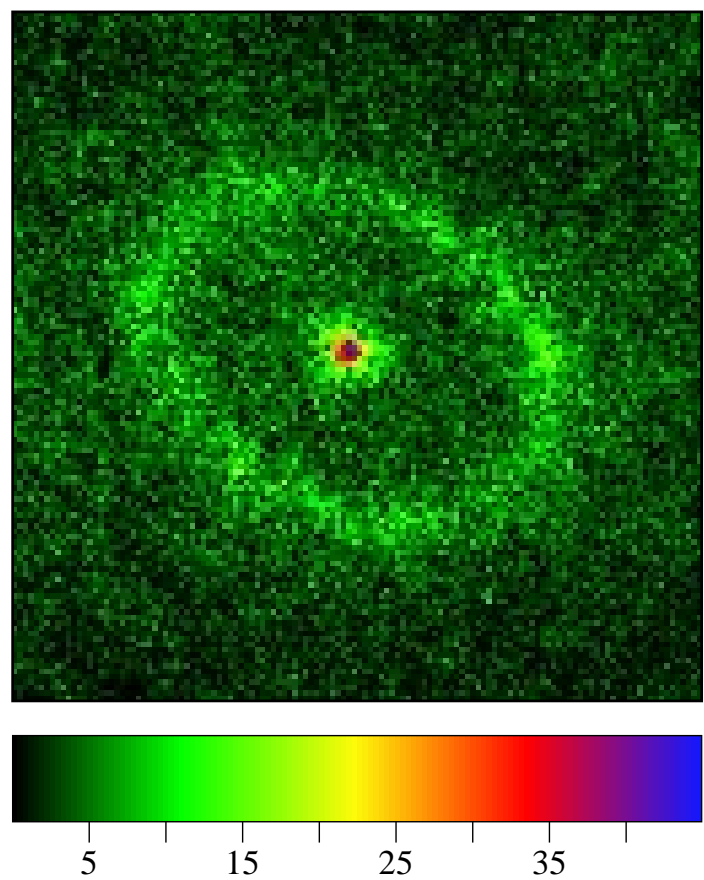

Fig 2.a: image of SN1987A.
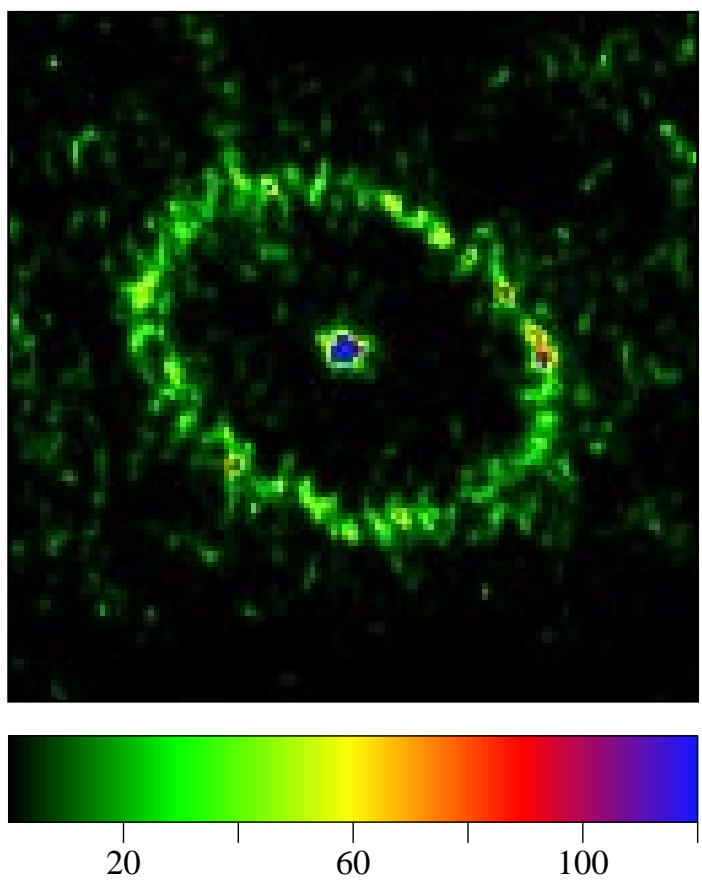

Fig 2.c: RLM deconvolution.
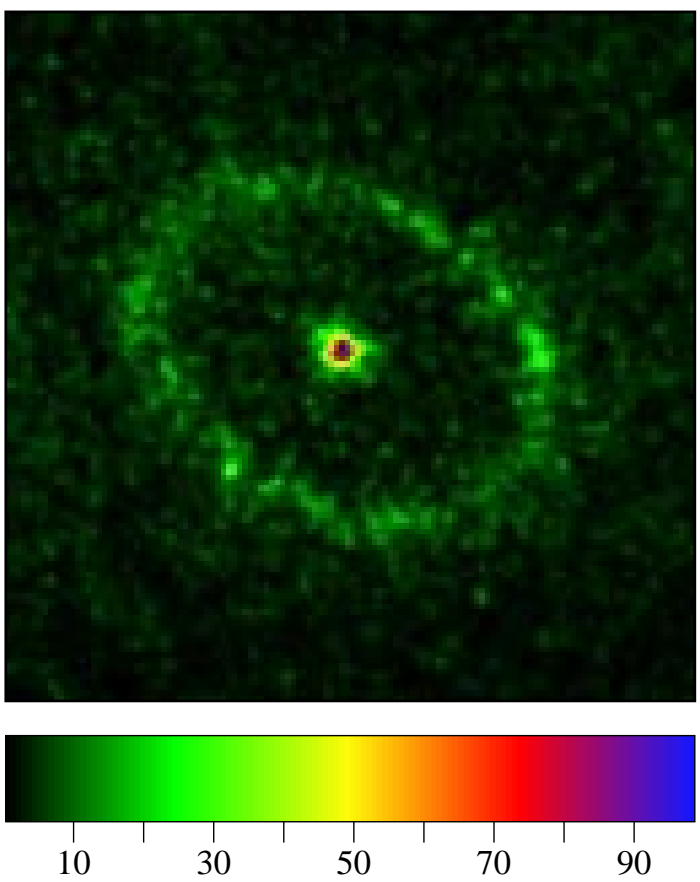

Fig 2.b: MEM deconvolution.
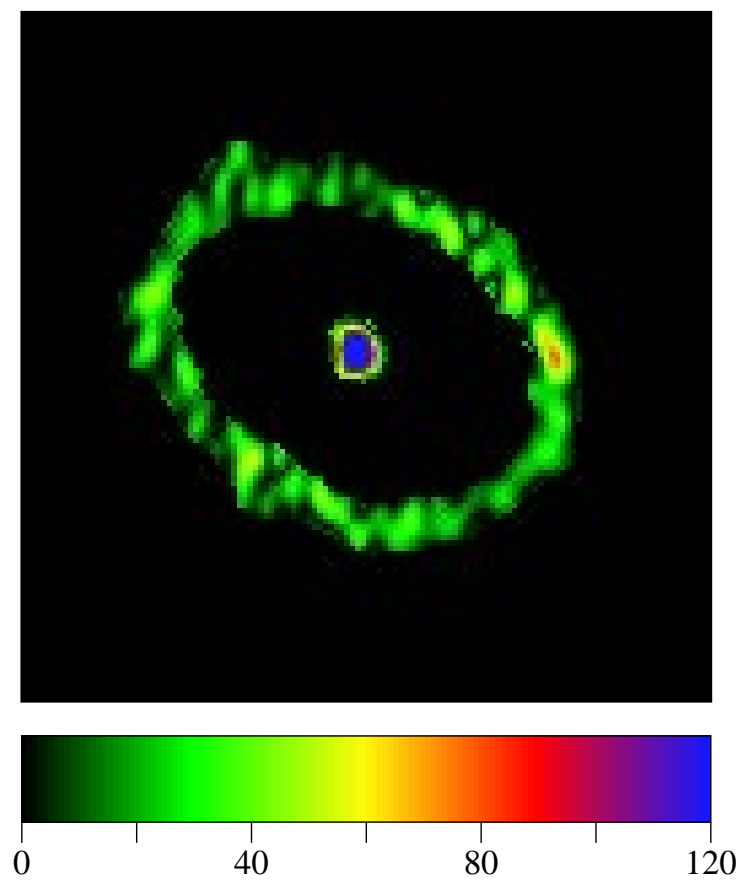

Fig 2.d: IDEA deconvolution.

Fig. 2. a) raw image of SN 1987A. b) IDEA deconvolution. c) MEM deconvolution. d) RLM deconvolution. The grayscale levels tables are below each figure. IDEA and RLM images are thresholded at the same levels. The images are $128 \times 128$ 


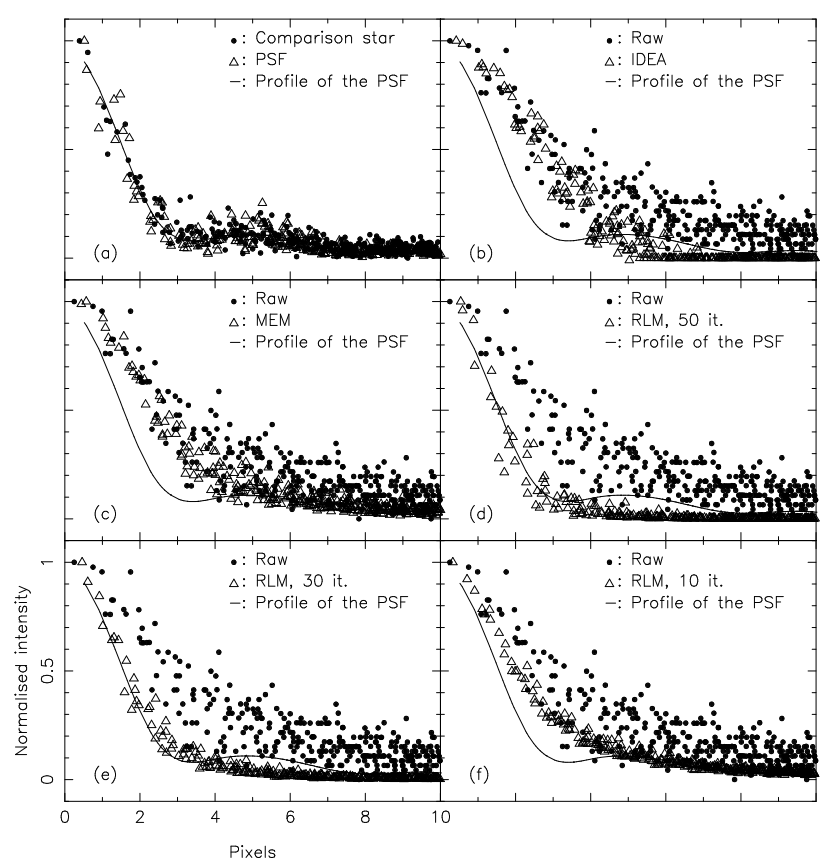

Fig. 3. a) radial profiles of the PSF and the star SE of SN 1987A. The curve is a polynomial fit to the PSF. On the other plots: profile of the PSF and profile of the supernova on the raw image compared with profiles of the supernova after the different deconvolutions (b) IDEA, c) MEM, d) RLM 50 iterations, e) RLM 30 iterations, f) RLM 10 iterations)

deconvolution, compared to other methods, is more reminiscent of an envelope around the star.

The total width of the bright structure of the ring of the supernova (Fig. 2) is $0{ }^{\prime \prime} 09$ with IDEA and $0 . \prime 08$ with RLM (50 iterations). This shows that this structure is slightly resolved and confirms that the "large" profile of the supernova seen on the IDEA solution is due to an envelope around the star.

On the IDEA solution, the ring is more filamentary and less noisy than on the other deconvolutions. It is rather blobby on the RLM solution suggesting some overresolution as shown in the analysis above. It is very noisy on the MEM solution probably because MEM fails to gather the information from the wings of the PSF which are at very low signal-to-noise ratio.

\section{2. $M 87$}

Because of the limited field of the FOC, two images are necessary to cover the entire jet. We call them "center image" (where the nucleus is present) and "jet image" (where the outer part of the jet is visible). They represent two different deconvolution problems because of the presence of a very bright unresolved source in the center image and a faint filamentary extended structure in the jet image. These images are presented in Boksenberg et al. (1992); they were obtained on April 5-6, 1991 and are
F220W exposures of 1197 s each. Here, the PSF is simulated with TINYTIM (simulation package of the HST's PSF, see Krist 1992).

In Figs. 4 and 5, we show the raw center image and the raw jet image, the RLM deconvolutions (40 iterations), the MEM deconvolutions (50 and 36 iterations respectively, with uniform noise of 0.69 and 1.0, poisson noise of 0.1 and 0.05 ) and the IDEA deconvolutions (gain in resolution of 1.8 and 1.7 with upper limit of $17.5 \%$ and $13 \%$ for the quadratic error; the solution is reached in 2 and 5 iterations respectively). The MEM solutions are clearly noisier and consequently miss faint details in the jet. The RLM solutions appear the most resolved, but as seen in SN 1987A analysis, the result may be biased by over-resolution. Moreover, the IDEA solutions do reveal the same level of detail while also showing fainter structures.

The presence of numerous artifacts (especially rings) around the center of the galaxy is due to the simulated PSF.

\section{3. $3 C 66 B$}

The HST observations of this jet through the F320W filter were first presented by Macchetto et al. (1991). In this work, we use images obtained through the F220W filter. Four $1197 \mathrm{~s}$ exposures were obtained on March 18, 1991. As the jet was barely visible on the individual frames, we averaged the four exposures to increase the signal-to-noise ratio. The PSF is simulated with TINYTIM as if it were for an individual frame and thus does not represent the exact PSF of the four exposures average.

In Fig. 6, we show the raw image, the RLM deconvolution (40 iterations), the MEM deconvolution (50 iterations, uniform noise of 4.6 and poisson noise of 0.2 ) and the IDEA deconvolution (gain in resolution of 1.8 with an upper limit of $18 \%$ for the quadratic error; the solution is reached after 2 iterations).

The MEM solution appears noisy and the jet is extremely weak. The RLM and the IDEA deconvolutions give very similar solutions; except that the B knot (brightest knot at about $2^{\prime \prime}$ from the nucleus, Fraix-Burnet et al. 1989) is filamentary on the IDEA deconvolution whereas it is blobby on the RLM one. This can be probably explained by over-resolution (see Sect. 3.1). In addition there are less artifacts around the center of the galaxy on the IDEA deconvolution than on the RLM deconvolution. The FWHM measured on the faint star North of the galaxy center is about $0{ }^{\prime \prime} 12$ for the raw, MEM, RLM and IDEA images. It is also the same for the center of $3 \mathrm{C} 66 \mathrm{~B}$ except for the RLM solution which has FWHM $\simeq 0$ ". 07 , indicating over-resolution on bright parts of the image. 

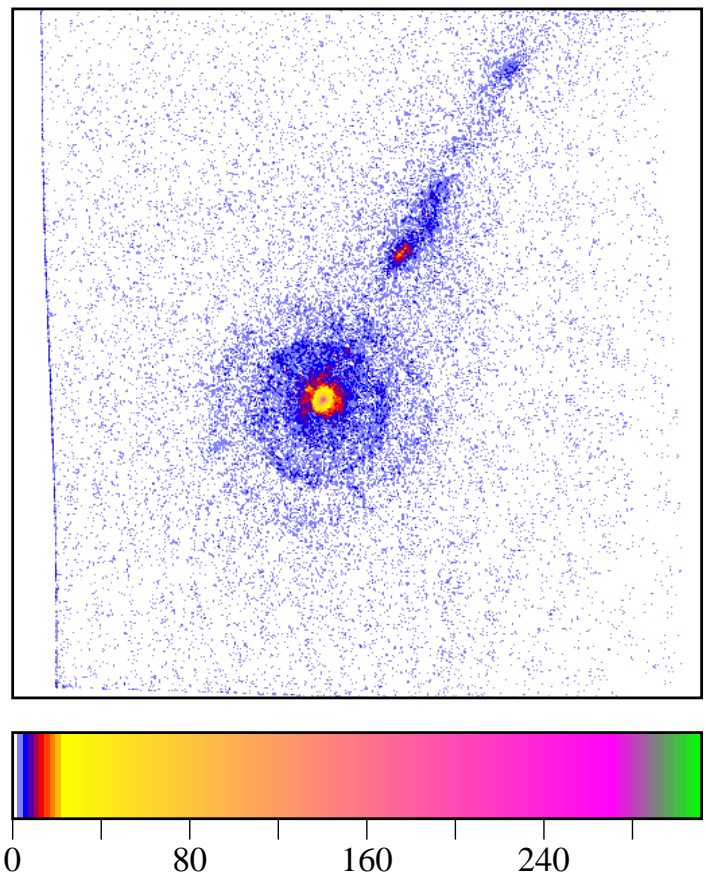

Fig 4.a: image of M87 center.
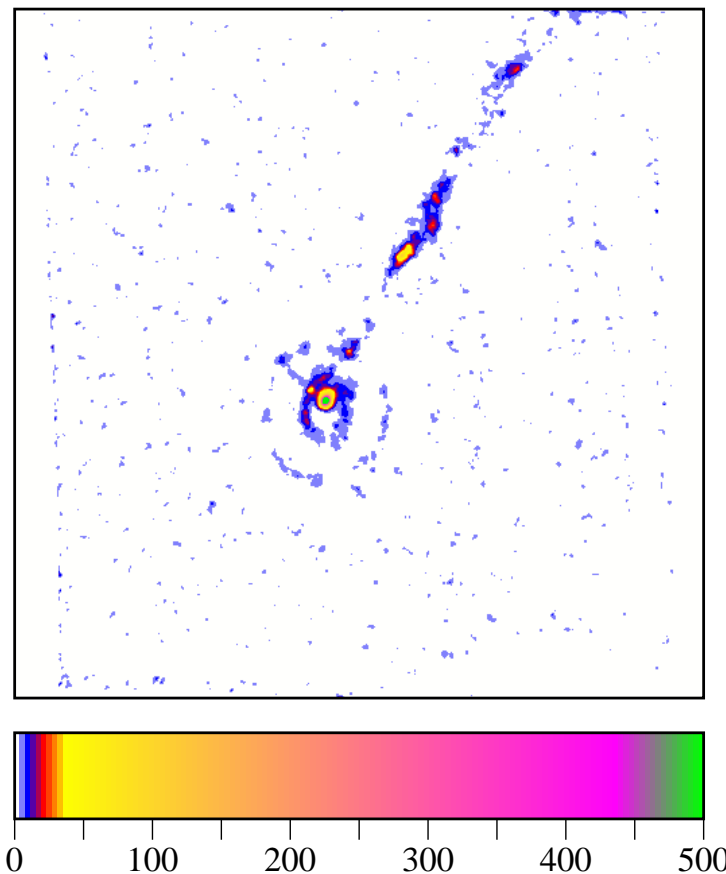

Fig 4.c: RLM deconvolution.
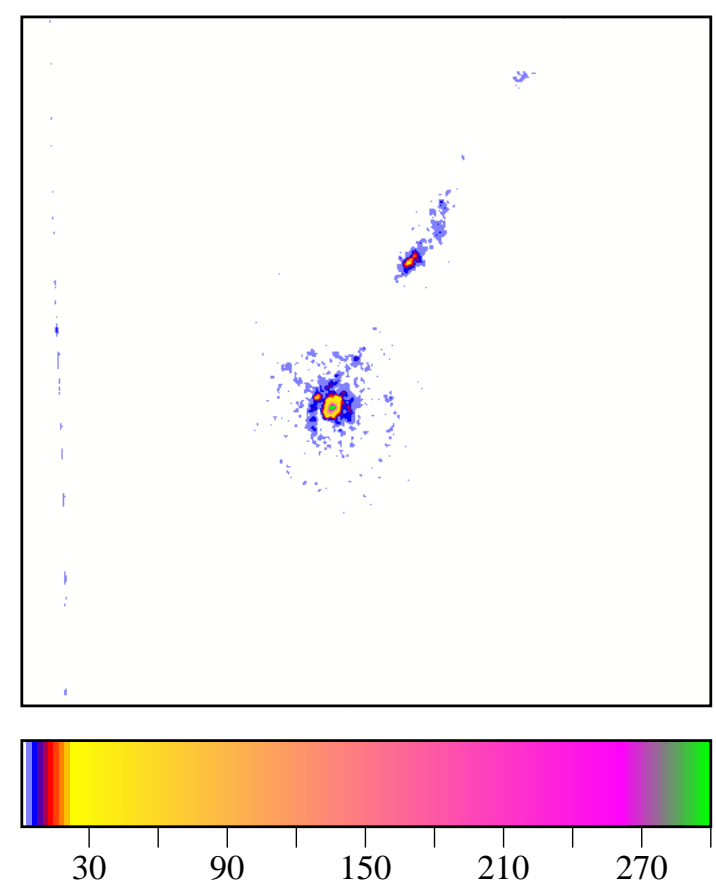

Fig 4.b: MEM deconvolution.
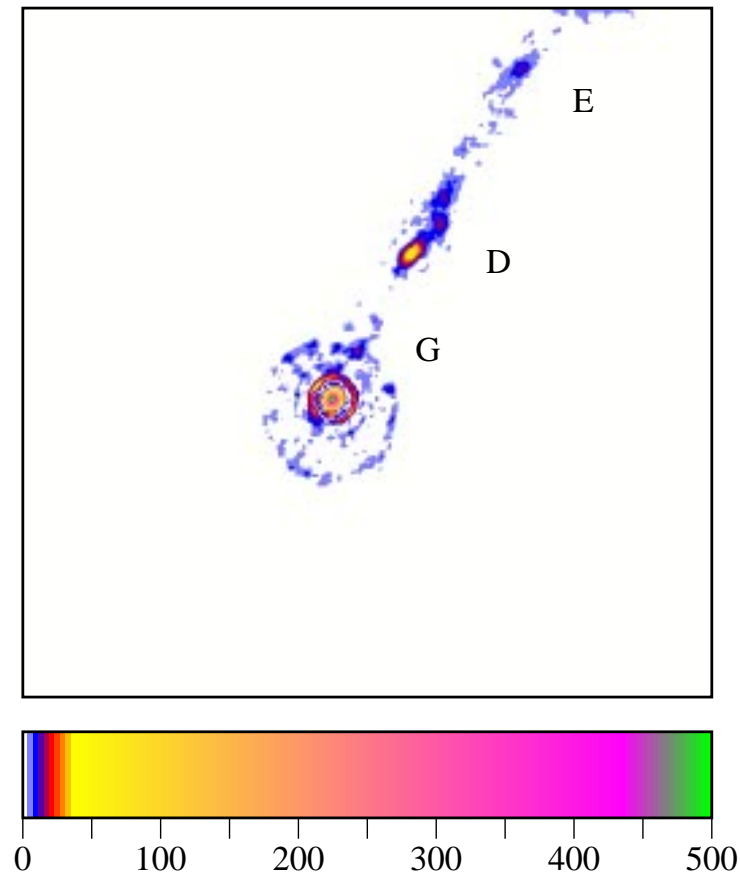

Fig 4.d: IDEA deconvolution.

Fig. 4. Same as Fig. 2 for the center of M 87. The images are $512 \times 512$. The PSF used for the deconvolutions is simulated using Tinytim 

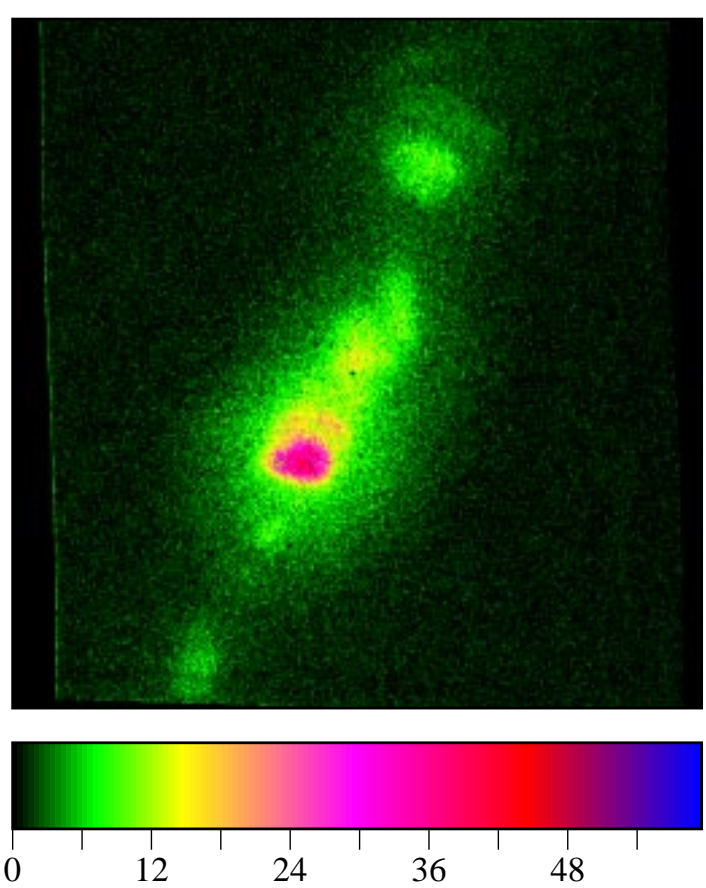

Fig 5.a: image of M87 jet.
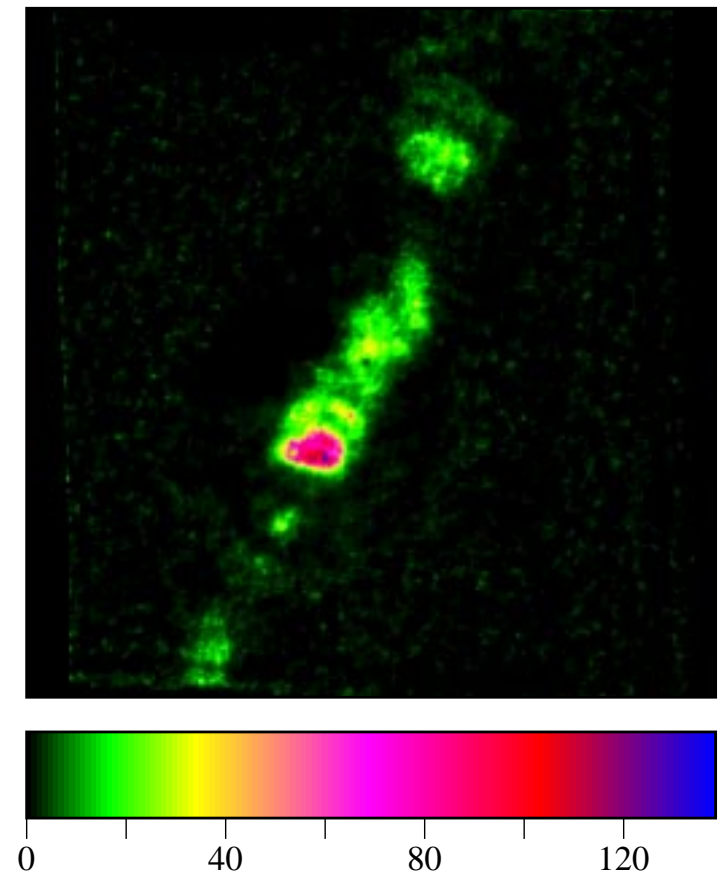

Fig 5.c: RLM deconvolution.
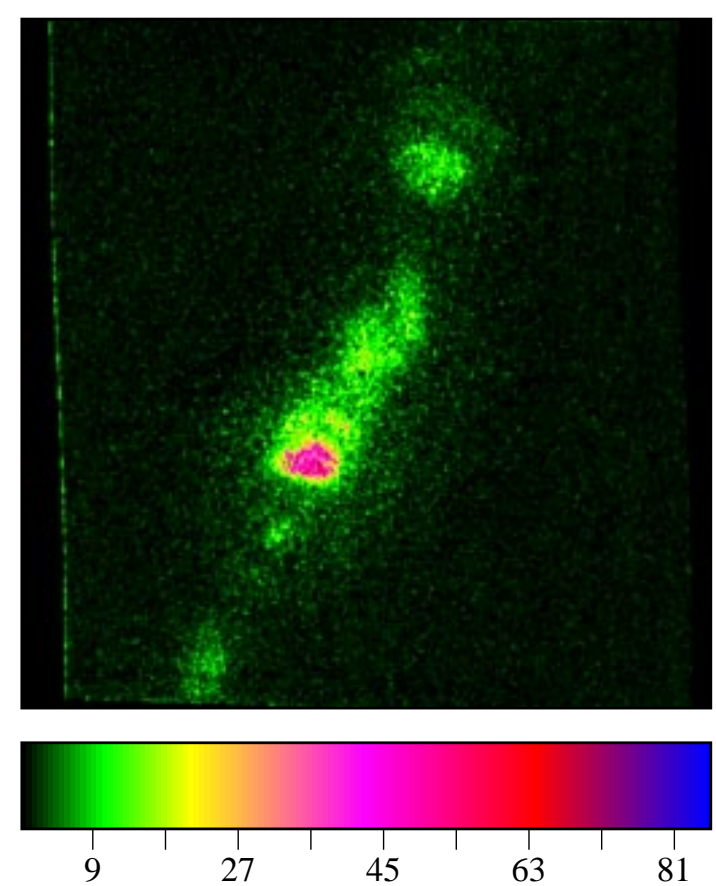

Fig 5.b: MEM deconvolution.
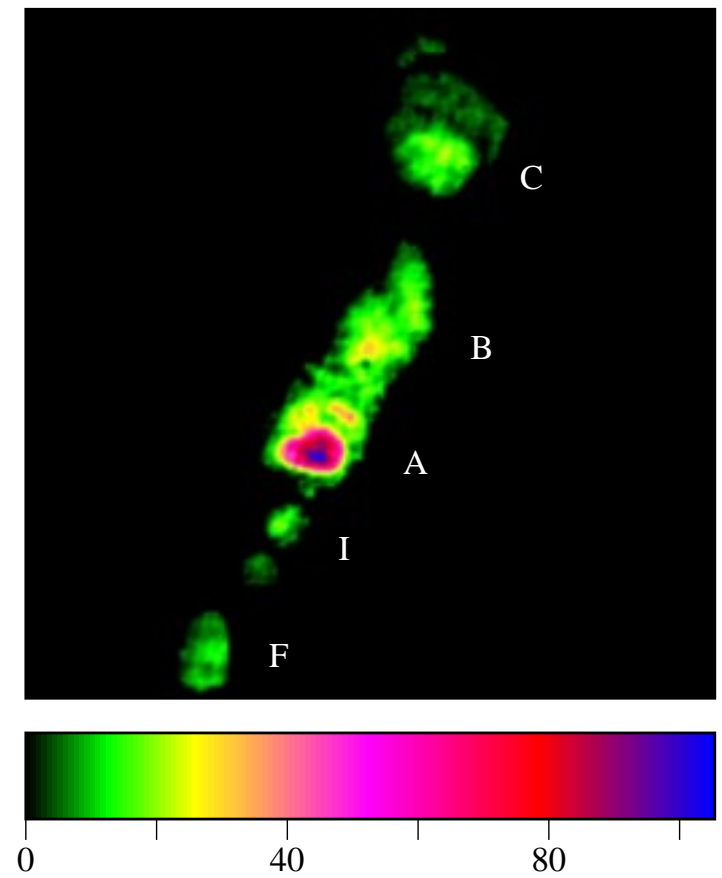

Fig 5.d: IDEA deconvolution.

Fig. 5. Same as Fig. 4 for the jet of $M 87$ 

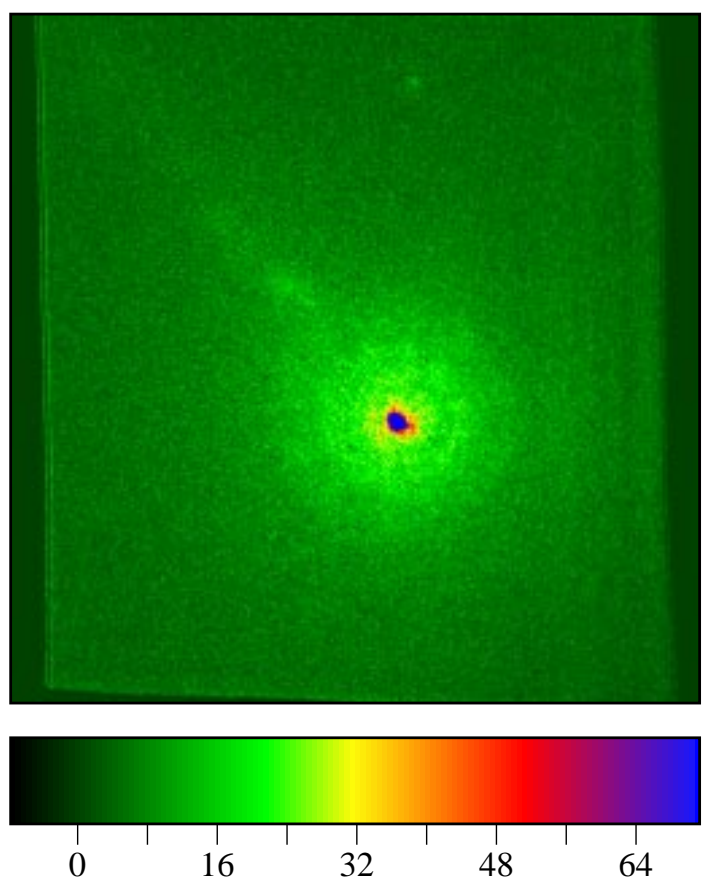

Fig 6.a: image of 3C66B jet.
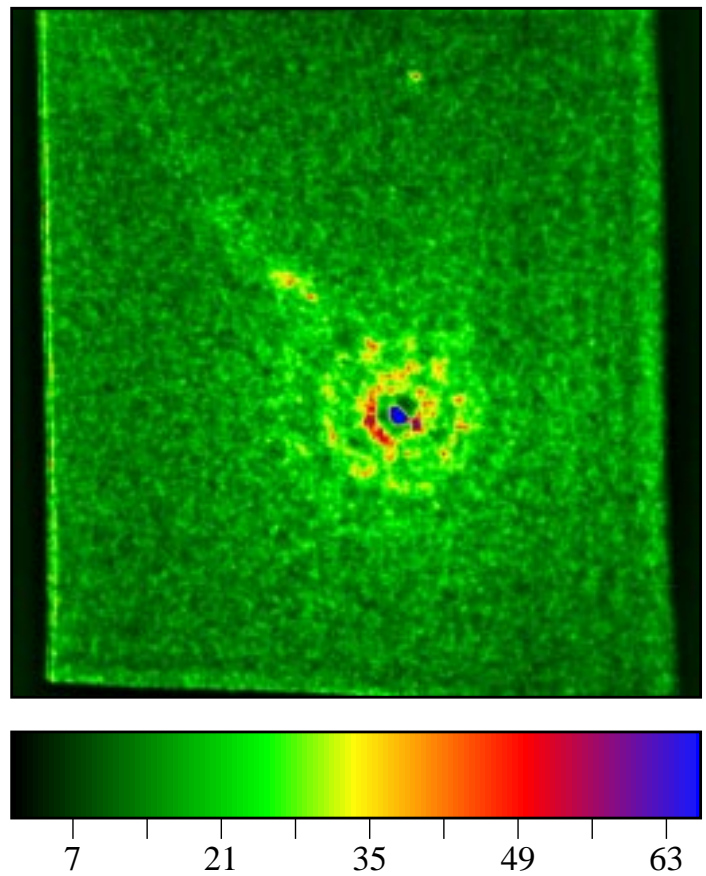

Fig 6.c: RLM deconvolution.

Fig. 6. Same as Fig. 4 for the jet of $3 \mathrm{C} 66 \mathrm{~B}$
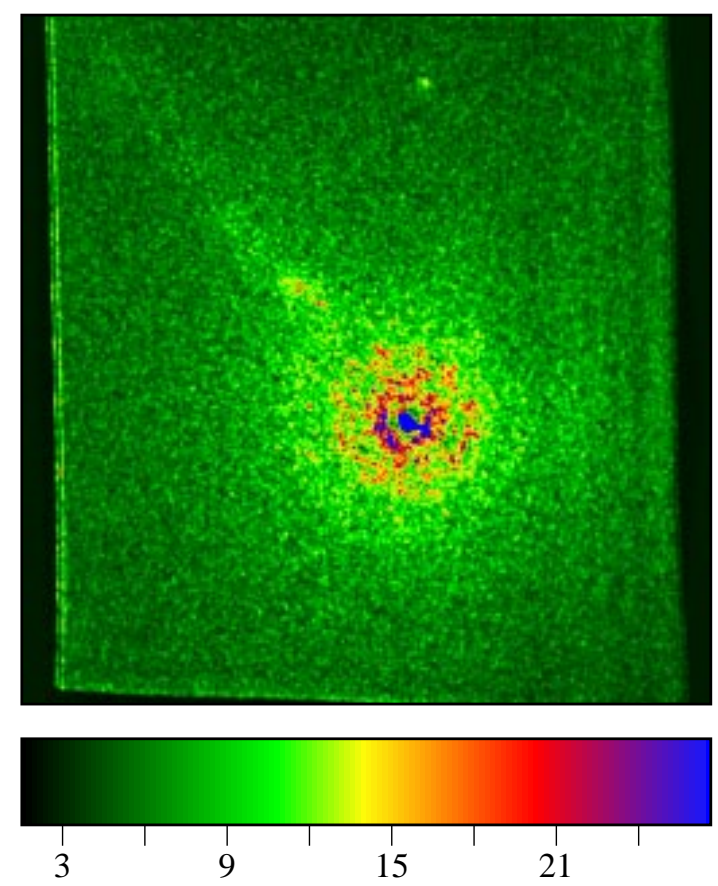

Fig 6.b: MEM deconvolution.
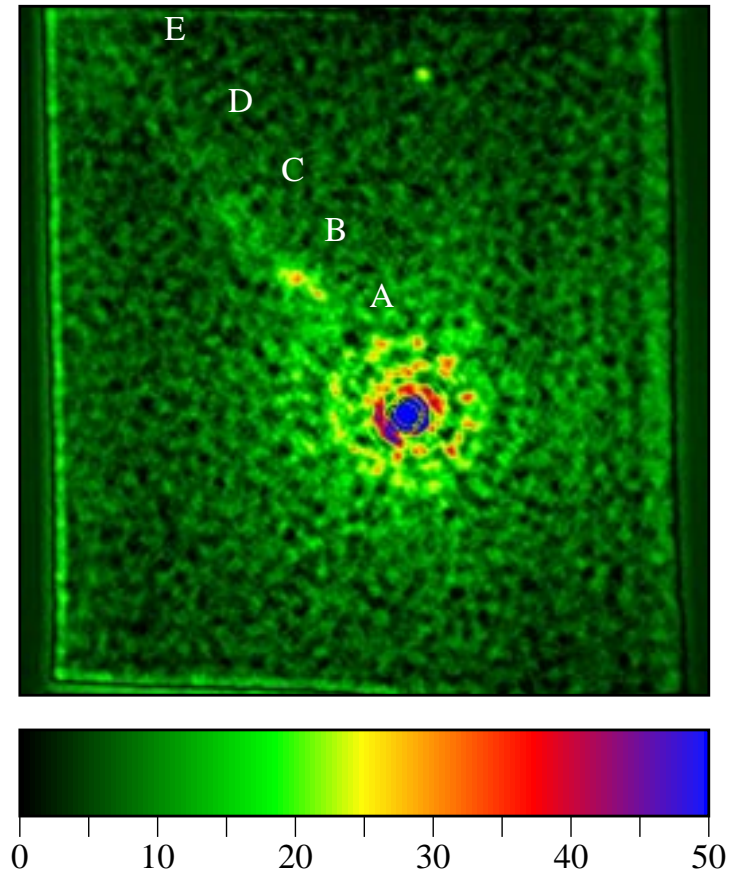

Fig 6.d: IDEA deconvolution. 


\section{Astrophysical considerations}

We consider in this section the photometric properties of the deconvolved images, except in the case of $3 \mathrm{C} 66 \mathrm{~B}$ for which a reliable calibration of the data is lacking.

\subsection{SN 1987 A}

The improvement in the quality of the deconvolved image prompts us to reconsider some results presented earlier (Jakobsen et al. 1991; Panagia et al. 1991). Interesting comparison can also be made with the work of Plait et al. (1995). They perform a RLM deconvolution, but on a F501N summed image, thus of higher signal-to-noise ratio than our single exposure image. Jakobsen et al. (1994) present also images obtained with the COSTAR corrected HST. Comparison will be made even though the structure and photometry of the ring has changed between the two observations.

The ratio of the intensity of the ring to the one inside could tell us whether it is really a ring or a shell (see Dwek $\&$ Felten 1992). Since we have shown that the RLM deconvolution artificially increases the intensity of the peaks in the ring, the ratio (between 10 and 100) found by Panagia et al. (1991) is thus probably overestimated. The IDEA solution indicates that this ratio might be in the range $30-40$, relaxing a little bit the constraint in favor of a ring. Moreover, Plait et al. (1995) argue that such a ratio results from a ring, and not from a shell.

The fluxes of the central star and of the ring has been measured within the support used in IDEA. For the raw image we used the values estimated by Jakobsen et al. (1991) for comparison. From Table 1 it is clear that IDEA recovers a large fraction of the energy from the wings of the PSF. Note that it is coherent with ground based observations obtained at the same epoch (see R. Cumming cited in Plait et al. 1995) giving a ring intensity of $1510^{-14} \mathrm{erg} \mathrm{s}^{-1} \mathrm{~cm}^{-2}$. In addition, the flux of the central star obtained with IDEA is compatible with a unique decay rate between days 1275 and 2522 (see Jakobsen et al. 1994).

Table 1. Absolute fluxes in $10^{-14} \mathrm{erg} \mathrm{s}^{-1} \mathrm{~cm}^{-2}$. The value for the raw image has been taken from Jakobsen et al. (1991)

\begin{tabular}{lll}
\hline Image & Center & Ring \\
\hline Raw & 5.9 & 30 \\
IDEA & 5.2 & 22 \\
RLM & 4.3 & 18 \\
MEM & 1.9 & 10 \\
\hline
\end{tabular}

The profile of the central star of the supernova presented in Sect. 3.1 shows the presence of an envelope around the star. This has already been found by Jakobsen et al. (1991). We measure similar values of the total radius for the envelope profile: 0.12 , that is (at a distance of $50 \mathrm{kpc}$ ) about 35 light-days, corresponding to an expansion speed of $\simeq 8000 \mathrm{~km} \mathrm{~s}^{-1}$. We also note that the central star on the IDEA image is elongated $\left(\mathrm{PA} \simeq 45^{\circ}\right)$. The elongation is absent on the other deconvolution images. This value is not very precise because of the anisotropy of the PSF, but is corroborated by the results with speckle interferometry providing an elongation with $\mathrm{PA}=20^{\circ}$ (Papaliolios et al. 1989) and with COSTAR-corrected FOC images by Jakobsen et al. (1994): $\mathrm{PA} \simeq 45^{\circ}$.

Panagia et al. (1991) noted the clumpiness of the light distribution in the ring. This might have three origins (Dwek \& Felten 1992): 1) anisotropy in the ionising photon propagation from the star to the ring; 2) clumpiness of the ambiant medium acting on the ring propagation; 3) heterogeneities within the ring (density or emissivity). On the RLM solution, the ring is really knotty, probably due to over-resolution. Further evidence is provided by the faint superposed star, located at $\mathrm{PA}=235^{\circ}$ on the ring, described in Plait et al. (1995). Its intensity with respect to that of the ring is artificially increased on the RLM image. On the opposite, the ring in the IDEA solution is rather continuous with a few regions (one especially) of increased brightness defining the "clumps". This shows that the ionising radiation is isotropic and that the ambiant medium has not disturbed the ring too much during its expansion. However, the south part of the ring is flatter than an ellipse, indicating probably a resistance of the ambiant medium. Some filaments in the ring (note the one to the East) suggest a certain level of heterogeneities. Note also how the northern and southern parts of the ring are brighter than the eastern and western parts. This is probably explained by the inclination of the ring with respect to the line of sight (Jakobsen et al. 1991). The relative smoothness of the ring found with IDEA is confirmed by the modelisation of the UV emission (Plait et al. 1995) which slightly favors the smooth ring model. The IDEA solution is close to the RLM deconvolution of Plait et al. (1995) (applied to a higher signal-to-noise ratio image). The IDEA image is also similar to the COSTAR-corrected images presented by Jakobsen et al. (1994) even though the ring structure might have evolved between the two epochs.

\section{2. $M 87$}

From the deconvolutions it can be seen that the center of the galaxy is elongated indicating the presence of a nuclear jet, confirming the statement of Boksenberg et al. (1992). However, the IDEA deconvolution suggests that the knot at $0.9^{\prime \prime}$ from the nucleus might be an artifact: it is less prominent on the IDEA deconvolution than on the RLM deconvolution and is exactly at the position of a ring created by the PSF. 
On the RLM deconvolution, knot D appears as 2 knots, whereas on the IDEA deconvolution it has the appearance of a filament. The filamentary structure of knot $\mathrm{E}$ is also more evident on the IDEA deconvolution. This is consistent with the general filamentation found in extragalactic jets (see Sect. 4.3 for another example). The continuity of the jet seems less obvious on the IDEA deconvolution, but there is a slight hint for a double filament in knot F. This is also true for the outer part of the jet (visible on the jet image). The IDEA features are essentially confirmed on WFPC2 images from HST (Ford et al. 1994).

Boksenberg et al. (1992) performed photometry on the raw image because of the lack of confidence in the deconvolution result. We use the IDEA solution after calibration of our raw data in the same manner. We present (Fig. 7) the profile of the jet obtained within perpendicular slits. Its width is defined by the support used for the deconvolution.

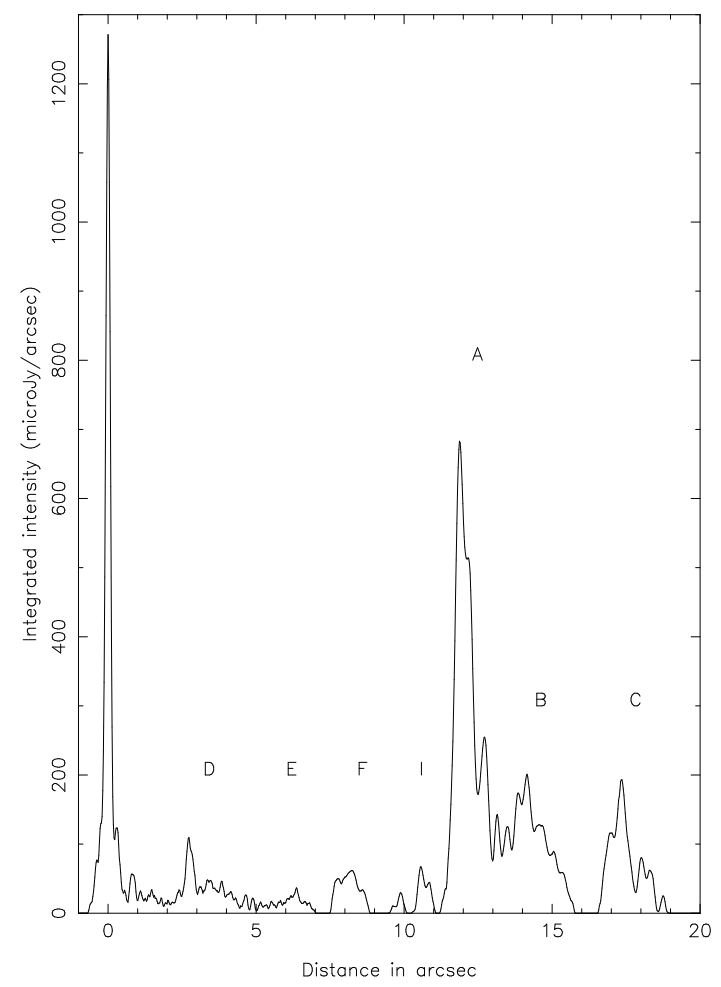

Fig. 7. Profile of the jet integrated over its whole width in a one-pixel-wide slit

This can be compared with Fig. 4 of Biretta et al. (1991) compiled using data at different wavelengths and a lower resolution. If we integrate this profile along the jet to have the total intensity of the knots, for the whole jet ( $1^{\prime \prime}$ to $19^{\prime \prime}$ from the nucleus) we find $1.2 \mathrm{mJy}$. From knots $\mathrm{F}$ to $\mathrm{C}$, our value $(1.1 \mathrm{mJy})$ is significantly higher than that found by Perola \& Tarenghi (1980) from IUE data
$(0.71 \pm 0.08 \mathrm{mJy})$. Perola's values are however compatible with ours knots A and B (0.81 mJy). That would indicate that knot $\mathrm{C}$ is not entirely in the IUE $10^{\prime \prime} \times 20^{\prime \prime}$ window.

\section{3. $3 C 66 B$}

The image presented in this paper is the first image of this jet at such a short wavelength. The jet is very faint, but the galaxy background is nearly absent making galaxy substraction unnecessary. As can be expected, the structure of the jet at this wavelength is identical to that at $320 \mathrm{~nm}$ (Macchetto et al. 1991), except may be for "the fatter structure" in which the double-stranded filament seems to be embedded. This could be naturally explained by the lower signal-to-noise ratio of the $220 \mathrm{~nm}$ image. We note that the B knot (Fraix-Burnet et al. 1989) appears to be filamentary on the IDEA deconvolution. This could indicate that the double-stranded filament is continuous from $2^{\prime \prime}$ from the nucleus outward. There is no trace of the "blue knots" found by Fraix-Burnet et al. (1989). But because of the artifacts left by the non-perfectly adapted PSF within about $2^{\prime \prime}$ from the nucleus, the detection of such faint features is certainly hopeless.

\section{Conclusion}

We have shown in this paper that deconvolution can bring a wealth of additional information even in a difficult case like the spherical aberration of HST and even when applied to low signal-to-noise ratio images. In many cases RLM yields over-resolution and MEM totally fails for signal-to-noise ratios lower than 10. Even if we apply more sophisticated version of MEM, like the Method of Maximum Entropy on the Mean (MMEM) (see Le Besnerais et al. 1995) the results are not drastically improved. IDEA has proven to be extremely powerful in two respects: control over error propagation and efficiency at low signal-to-noise ratios. Both points are essential to astronomers. Moreover, the approach of IDEA allows us an interactive choice for the compromise between gain in resolution and stability of the solution. As to CPU time, the three codes are comparable. IDEA usually converges in a lower number of iterates but each one lasts longer (involving an iterate of the conjugate gradients method).

The main astrophysical result of this study is that for all images (the ring of SN 1987A or the jets of M 87 and $3 \mathrm{C} 66 \mathrm{~B})$ IDEA preserves the filamentary aspects of the objects and does not generate artificial "blobs".

We would like to emphasize that IDEA has been developed independently from the spherical aberration of the HST, so that it can be used on any kind of images. Indeed, even the COSTAR-corrected HST could still benefit from deconvolution. In addition improvements of the method are in progress. For instance, using orthogonal bases of compactly supported wavelets, Roques et al. (1996) have incorporated, in the wavelet domain, the 
Donoho \& Johnstone denoising method (Donoho 1992), which consists in a non-linear shrinkage of the wavelet coefficients. This allows us to recover the denoised image, whose Fourier transform, compared to raw Fourier data, yields the pointwise spectral signal-to-noise ratio which explicitly occurs in the formulation of the regularization function $g$. The advantages of this procedure are that the noise is almost entirely suppressed, and features sharp in the original remain sharp in the denoised image.

Since we could not render in grayscale levels Figs. 2a to $6 \mathrm{~d}$ a postscript color version of these pages is made available via "anonymous" FTP from:

$<\mathrm{ftp}: / /$ ftp.cerfacs.fr/pub/dsp/articles/AA $>$.

\section{References}

Biretta J.A., Stern C.P., Harris D.E., 1991, AJ 101, 1632

Boksenberg A., et al., 1992, A\&A 261, 393

Bouyoucef K., Fraix-Burnet D., Roques S., 1994, in: The Restoration of HST Images and Spectra II, Hanish R.J., White R.L. (eds.), Space Telescope Science Institute, p. 64 Daubechies I., 1992, Ten lectures on wavelets, SIAM

Donoho D.L., 1992, "De-noising by soft-thresholding", Technical report, Stanford University

Dwek E., Felten J.E., 1992, ApJ 387, 551

Ford H.C., Harms R.J., Tsetanov Z.I., et al., 1994, ApJ 435, L27

Fraix-Burnet D., Nieto J.-L., Lelièvre G., et al., 1989, ApJ 336, 121
Jakobsen P., et al., 1991, ApJ 369, L63

Jakobsen P., Jedrzejewski R., Macchetto F., Panagia N., 1994, ApJ 435, L47

Krist J., 1992, The Tinytim manual

Lannes A., Roques S., Casanove M.J., 1987a, J. Mod. Opt. 34, 161

Lannes A., Casanove M.J., Roques S., 1987b, J. Mod. Opt. 34, 321

Lannes A., Roques S., Casanove M.J., 1987c, J. Opt. Soc. Am. 4, 189

Lannes A., Anterrieu E., Bouyoucef K., 1996, to appear J. Mod. Opt.

Le Besnerais G., Bercher J.F., Demoment G., 1995 (submitted to IEEE Trans. on Inf. Th.)

Lucy L.B., 1974, AJ 79, 745

Macchetto F., et al., 1991, ApJ 373, L55

Mallat S., 1989, IEEE Trans. Pat. An. Mach. Int. 11, 674

Narayan R., Nityananda R., 1986, ARA\&A 24, 127

Panagia N., Gilmozzi R., Macchetto F., Adorf H.-M., Kirshner R.P., 1991, ApJ 380, L23

Papaliolios C., Karovska M., Koechlin L., Nisenson P., Standley C., Heathcote S., 1989, Nat 338, 565

Perola G.C., Tarenghi M., 1980, ApJ 240, 447

Plait P.C., Lundqvist P., Chevalier R.A., Kirshner R.P., 1995, ApJ 439, 730

Roques S., Bourzeix F., Bouyoucef K., 1996 (to appear in Exp. Astron.)

Slepian D., Polack H.O., 1961, Bell Syst. Tech. J., pp. 43-63

Tikhonov A.N., Arsenin V.Y., 1977, Solutions of ill-posed problems, Winston, Washington DC 